PROCEEDINGS OF THE

AMERICAN MATHEMATICAL SOCIETY

Volume 127, Number 7, Pages 2143-2146

S 0002-9939(99)05187-4

Article electronically published on March 16, 1999

\title{
ON THE SEMISIMPLICITY OF POLYHEDRAL ISOMETRIES
}

\author{
MARTIN R. BRIDSON
}

(Communicated by Ronald M. Solomon)

\begin{abstract}
If a polyhedral complex $K$ has only finitely many isometry types of cells, then all of its cellular isometries are semisimple. If $K$ is 1-connected and non-positively curved, then any solvable group that acts freely by cellular isometries on $K$ is finitely generated and contains an abelian subgroup of finite index.
\end{abstract}

In this note I shall prove that if a metric polyhedral complex has only finitely many isometry types of cells, then all of its cellular isometries are semisimple (Theorem A). This theorem is a consequence of the results in the first chapter of my thesis [B], and I ought to have made it explicit at the time. While correcting this oversight, I shall give an application that indicates the utility of Theorem A.

Given an isometry $\gamma$ of a metric space $X$, let $|\gamma|=\inf \{d(x, \gamma \cdot x) \mid x \in X\}$. One says that $\gamma$ is semisimple if there exists $x_{0} \in X$ such that $d\left(x_{0}, \gamma \cdot x_{0}\right)=|\gamma|$. Following [B], we write Shapes $(K)$ to denote the set of isometry classes of cells in a metric polyhedral complex.

Theorem A. Let $K$ be a connected $M_{\kappa}$-polyhedral complex. If Shapes $(K)$ is finite, then every cellular isometry of $K$ is semisimple.

Theorem B. If $K$ is a 1-connected, non-positively curved, $M_{\kappa}$-polyhedral complex with Shapes $(K)$ finite, and if $\Gamma$ is a solvable group that acts freely by cellular isometries on $K$, then $\Gamma$ is finitely generated and contains an abelian subgroup of finite index. (The torsion-free rank of this abelian subgroup is no greater than the dimension of $K$.)

Theorem B does not extend to the case of semisimple actions on complete, 1connected, non-positively curved complexes in which Shapes $(K)$ is not finite. For instance the additive rationals act freely and semisimply on a 2-dimensional complex of this type [BH, II.7].

In the case where $K$ is a smooth manifold, Theorem B is due to Gromoll and Wolf [GW] (see also Lawson and Yau [LY]). Gromov [G2] was the first to point out that the results of $[\mathrm{GW}]$ remain valid beyond the setting of Riemannian manifolds.

The elements of Theorems A and B can be found scattered in my book with André Haefliger $[\mathrm{BH}]$. I was motivated to write this more concise and explicit account by an interesting lecture which Jacek Świątkowski gave at the Banach

Received by the editors October 7, 1997.

1991 Mathematics Subject Classification. Primary 53C23, 20F32.

Key words and phrases. Non-positive curvature, semisimple isometries.

This work was supported by an EPSRC Advanced Fellowship, NSF grant 9401362 and the British Council. 
Centre in Warsaw in September 1997. In that lecture he described his recent work with Werner Ballmann on foldable cube complexes [BS], where a special case of Theorem A plays a significant rôle.

\section{CompleXes with Shapes $(K)$ Finite}

We recall what it means to say that $K$ is an $M_{\kappa}$-polyhedral complex with $\operatorname{Shapes}(K)$ finite. (We refer the reader to [BH, I.7] for a more detailed treatment.)

First one has the set of isometry types of cells, denoted Shapes $(K)$, which are convex polyhedral cells in $M_{\kappa}^{n}$, the complete 1-connected manifold of constant sectional curvature $\kappa$. The complex $K$ is formed by taking the disjoint union of (perhaps infinitely many) copies of the members of Shapes $(K)$ and then gluing them along isometric faces. It is not assumed that $K$ is locally compact: infinitely many cells may intersect in a given face.

It is proved in [B] that if $K$ is connected and $\operatorname{Shapes}(K)$ is finite, then one can make $K$ into a complete geodesic space by defining the distance between two points to be the infimum of the lengths of the paths connecting them, where length is measured in the given metrics on the individual cells. This metric is called the intrinsic metric on $K$ and will be denoted by $d_{K}$. Also, for each closed cell $S \subset K$ we fix an isometry $f_{S}: \bar{S} \rightarrow S$ where $\bar{S} \in \operatorname{Shapes}(K)$.

The proof of the existence of geodesics given in [B] also establishes the following fact, which is the key to Theorem A (see [B, 1.11] or [BH, I.7.30]).

Lemma 1. If Shapes $(K)$ is finite, then there exists a constant $\beta>0$ such that every geodesic segment $c \subset K$ is contained in a subcomplex $L_{c} \subset K$ that is the union of less than $\beta \ell(c)+1$ closed cells, where $\ell(c)$ is the length of $c$.

Given an $M_{\kappa}$-polyhedral complex $K$, model cells $\bar{S}, \bar{S}^{\prime} \in \operatorname{Shapes}(K)$ and points $\bar{x} \in \bar{S}$ and $\bar{y} \in \bar{S}^{\prime}$, we consider the sets of points in $K$ that lie above $\bar{x}$ and $\bar{y}$ :

$$
\begin{aligned}
X_{K} & =\{x \in K \mid \exists \text { isometry } f \text { from } \bar{S} \text { to a closed cell in } K \text { with } f(\bar{x})=x\} \text { and } \\
Y_{K} & =\left\{y \in K \mid \exists \text { isometry } g \text { from } \bar{S}^{\prime} \text { to a closed cell in } K \text { with } g(\bar{y})=y\right\} .
\end{aligned}
$$

Lemma 2. If Shapes $(K)$ is finite, then $\left\{d_{K}(x, y) \mid x \in X_{K}, y \in Y_{K}\right\} \subset[0, \infty)$ is discrete.

Proof. The number of points of $X_{K}$ or $Y_{K}$ that lie in any given closed cell of $K$ is bounded by the maximum of the orders of the isometry groups of the model cells $\bar{S} \in \operatorname{Shapes}(K)$. Hence, for each finite subcomplex $L \subset K$, the set of numbers $\left\{d_{L}(x, y) \mid x \in X_{K} \cap L, y \in Y_{K} \cap L\right\}$ is finite, where $d_{L}$ denotes the intrinsic metric on $L$ (not the restriction of $\left.d_{K}\right)$. Note that $d_{K}(x, y) \leq d_{L}(x, y)$ with equality if and only if some geodesic joining $x$ to $y$ in $K$ is contained in $L$.

Given $N \in \mathbb{N}$, we let $\Sigma_{N}=\bigcup\left\{d_{L}(x, y) \mid x \in X_{K} \cap L, y \in Y_{K} \cap L\right\}$, where the union is taken only over those subcomplexes $L \subset K$ which can be expressed as the union of at most $N$ closed cells; there are only finitely many such subcomplexes up to cellular isometry, so $\Sigma_{N}$ is finite.

According to Lemma 1 , for every $\ell>0$ there exists an integer $N_{\ell}$ such that every geodesic in $K$ of length less than $\ell$ is contained in a subcomplex which is the union of at most $N_{\ell}$ cells. Thus $\left\{d_{K}(x, y) \mid x \in X_{K}, y \in Y_{K}\right\} \cap[0, \ell) \subset \Sigma_{N_{\ell}}$ is finite. 


\section{Proof of Theorem A}

Suppose that Shapes $(K)$ is finite and let $\gamma$ be a cellular isometry of $K$. We choose a sequence of points $x_{n} \in K$ such that $d\left(\gamma \cdot x_{n}, x_{n}\right) \rightarrow|\gamma|$ as $n \rightarrow \infty$. Let $S_{n}$ be a closed cell containing $x_{n}$. Because Shapes $(K)$ is finite, by passing to a subsequence we may assume that all of the $\bar{S}_{n}$ are the same, equal to $\bar{S}$ say. Let $\bar{x}_{n} \in \bar{S}$ be the point such that $f_{S_{n}}\left(\bar{x}_{n}\right)=x_{n}$. By passing to a further subsequence we may assume that the sequence $\bar{x}_{n}$ converges, to $\bar{x} \in \bar{S}$ say, as $n \rightarrow \infty$.

$$
d_{K}\left(\gamma \cdot f_{S_{n}}(\bar{x}), f_{S_{n}}(\bar{x})\right) \leq d_{K}\left(\gamma \cdot f_{S_{n}}(\bar{x}), \gamma \cdot x_{n}\right)+d_{K}\left(\gamma \cdot x_{n}, x_{n}\right)+d_{K}\left(x_{n}, f_{S_{n}}(\bar{x})\right) .
$$

By assumption, the second term on the right tends to $|\gamma|$, and since we obviously have $d_{K}\left(x_{n}, f_{S_{n}}(\bar{x})\right) \leq d_{\bar{S}}\left(\bar{x}_{n}, \bar{x}\right)$, which tends to 0 , we see that the righthand side of the above inequality tends to $|\gamma|$ as $n \rightarrow \infty$. But the value of the term on the left cannot be less than $|\gamma|$, so since it has to lie in a discrete set $^{1}$ (Lemma 2) we deduce that $d_{K}\left(\gamma \cdot f_{S_{n}}(\bar{x}), f_{S_{n}}(\bar{x})\right)=|\gamma|$ when $n$ is sufficiently large.

In the next section we shall need the following companion to Theorem A.

Proposition. Let $K$ be an $M_{\kappa}$-polyhedral complex with Shapes $(K)$ finite. If $\Gamma$ acts by cellular isometries on $K$, then $\{|\gamma|: \gamma \in \Gamma\} \subset[0, \infty)$ is discrete.

Proof. Fix $\ell>0$. Suppose $|\gamma| \leq \ell$ and let $x \in K$ be such that $d(\gamma \cdot x, x)=|\gamma|$. Let $S$ be a closed cell containing $x$. By Lemma 1, there is an integer $N$ depending only on $\ell$ and Shapes $(K)$ such that there exists a subcomplex $L \subset K$ that consists of at most $N$ closed cells, contains $S$ and $\gamma . S$, and contains a geodesic joining $x$ to $\gamma \cdot x$. $d_{K}(x, \gamma \cdot x)=\min \left\{d_{L}(y, \gamma \cdot y) \mid y \in S\right\}$, which depends only on the isometry type of $L$, a choice of two cells from $L$, and a choice of isometry between these cells. Given $\ell$, there are only finitely many such choices.

\section{Proof of Theorem B}

Let $K$ and $\Gamma$ be as in the statement of Theorem B. Following Gromov [G2], we use the term $\operatorname{CAT}(0)$ space to mean a 1-connected geodesic metric space that is non-positively curved in the sense of A.D. Alexandrov.

The Flat Torus Theorem [BH, II.7] implies that if $H \cong \mathbb{Z}^{n}$ acts freely by semisimple isometries on a complete CAT(0) space $X$, then there is an $H$-invariant isometrically embedded $m$-flat $\mathbb{E}^{m} \hookrightarrow X$, where $m \leq n$ and each $h \in H$ acts on this flat as a translation of amplitude $|h|$. If $m<n$, then $\{|h|: h \in H\}$ accumulates at 0 . So by the Proposition, if $X=K$ and $H \subset \Gamma$, then $m=n \leq \operatorname{dim} K$, and hence there is a bound on the rank of free abelian subgroups in $\Gamma$.

It also follows from the Proposition that $\Gamma$ does not contain any infinitely ascending chain of free abelian subgroups of a fixed rank. And because it acts freely, $\Gamma$ is torsion-free [BH, II.2]. Therefore all abelian subgroups of $\Gamma$ are finitely generated. But if all of the abelian subgroups of a solvable group are finitely generated, then the group is polycyclic [Se]. Thus we have reduced to the problem of showing that if a polycyclic group acts discretely by semisimple isometries on a complete CAT $(0)$ space, then the group is virtually abelian.

Arguing by induction on the Hirsch length of the group, one sees that a polycyclic group is virtually abelian if and only if it does not contain a subgroup of the form $\mathbb{Z}^{n} \rtimes_{\phi} \mathbb{Z}$ with $\phi \in G L_{n}(\mathbb{Z})$ of infinite order. In such a subgroup, infinitely many

\footnotetext{
${ }^{1}$ This is the only point at which we use the fact that the action of $\Gamma$ is by cellular isometries.
} 
distinct elements of the normal $\mathbb{Z}^{n}$ are conjugate. The translation length $|\gamma|$ of an isometry of a $\mathrm{CAT}(0)$ space is a conjugacy invariant, so if $\mathbb{Z}^{n} \rtimes_{\phi} \mathbb{Z}$ were to act freely and discretely by semisimple isometries on a CAT(0) space, then in the discrete action of $\mathbb{Z}^{n}$ on the $n$-flat yielded by the Flat Torus Theorem, infinitely many elements of $\mathbb{Z}^{n}$ would act as translations of the same amplitude, which is clearly impossible.

Remark. Instead of assuming that the action of $\Gamma$ is free, one can assume that the isotropy subgroups of the action are finite and of bounded order.

\section{ACTIONS OF LATTICES}

We conclude with a remark explaining how Theorem B gives rise to a fixed point theorem for certain actions of lattices on polyhedral complexes.

Let $G$ be a semisimple Lie group with finite centre and no compact factors. If $\mathbb{R}$-rank $(G) \geq 2$, then any non-uniform, irreducible lattice $\Gamma \subset G$ contains a solvable subgroup that is not virtually abelian [M]. It follows from Theorem B that if $K$ is a non-positively curved, 1-connected $M_{\kappa}$-polyhedral complex with $\operatorname{Shapes}(K)$ finite, and $\Psi: \Gamma \rightarrow \operatorname{Isom}(K)$ is an action by cellular isometries, then ker $\Psi$ is infinite. But the Margulis-Kazhdan Finiteness Theorem ([Z, 8.1.2]) states that every normal subgroup of $\Gamma$ is either finite or of finite index. Thus the image of $\Psi$ must be finite. The intrinsic metric on $K$ is complete [B] and any finite group of isometries of a complete CAT(0) space has a fixed point [BH, II.2], therefore the action of $\Gamma$ fixes a point of $K$.

\section{REFERENCES}

[B] M.R. Bridson, Geodesics and curvature in metric simplicial complexes, Group Theory From a Geometrical Viewpoint (E. Ghys, A. Haefliger, A. Verjovsky, ed.), World Scientific, 1991, pp. 373-463. MR 94c:57040

[BH] M.R. Bridson and A. Haefliger, Metric spaces of non-positive curvature, Grundlehren der Mathematischen Wissenschaften, Springer-Verlag, Berlin-Heidelberg-New York, 1999.

$[\mathrm{BM}] \mathrm{M}$. Burger and S. Mozes, CAT(-1) spaces, divergence groups and their commensurators, J. Amer. Math. Soc. 9 (1996), 57-93. MR 96c:20065

[BS] W. Ballmann and J. Świạtkowski, On groups which act on cube complexes, preprint, 1998.

[GW] D. Gromoll and J. Wolf, Some relations between the metric structure and the algebraic structure of the fundamental group in manifolds of nonpositive curvature, Bull. Amer. Math. Soc. 77 (1971), 545-552. MR 43:6841

[G1] M. Gromov, Hyperbolic groups, Essays in group theory (S.M. Gersten, ed.), MSRI Publication 8, Springer-Verlag, New York, 1988, pp. 75-263. MR 89e:20070

[G2] M. Gromov, Hyperbolic manifolds, groups and actions, Riemann Surfaces and related Topics (Stony Brook, 1978), Ann. of Math. Studies, vol. 97, Princeton Univ. Press, 1981, pp. 183213.

[LY] H. B. Lawson and S. T. Yau, Compact manifolds of nonpositive curvature, J. Diff. Geom. 7 (1972), 211-228. MR 48:12402

[M] G. A. Margulis, Discrete subgroups of Lie groups, Ergebnisse der Math., Springer-Verlag, Berlin-Heidelberg-New York, 1990.

[Se] D. Segal, Polycyclic groups, Camb. Univ. Press, 1985. MR 85h:20003

[Sr] J-P. Serre, Trees, Springer-Verlag, Berlin-Heidelberg-New York, 1980. MR 82c:20083

[Z] R. Zimmer, Ergodic Theory and Semisimple Groups, Monog. Math., Vol 81, Birkhäuser, Basel, 1984. MR 86j:22014

Mathematical Institute, 24-29 St Giles', Oxford, OX1 3LB, United Kingdom

E-mail address: bridson@maths.ox.ac.uk 